\title{
Right Lung
}

National Cancer Institute

\section{Source}

National Cancer Institute. Right Lung. NCI Thesaurus. Code C33483.

The 3-lobed lung located on the right side of the body. 